\title{
On the Optimality of Treating Interference as Noise for $K$-user Compound Interference Channels
}

\author{
Chunhua Geng, and Syed A. Jafar \\ Center for Pervasive Communications and Computing (CPCC), University of California Irvine, Irvine, CA 92697 \\ Email: \{chunhug, syed\}@uci.edu
}

\begin{abstract}
For the $K$-user interference channel, Geng et al. identify a general condition under which power control and treating interference as noise (TIN) is optimal from the perspective of generalized degrees of freedom (GDoF). In this work, we show that for a $K$-user compound interference channel, if in every possible state for each receiver, the channel satisfies the TIN-optimality condition of Geng et al., then power control and TIN achieves the entire GDoF region of the compound channel. For an arbitrary compound interference channel, we find a nontrivial counterpart regular interference channel, such that the two have the same TIN region, and the GDoF-optimal power control problems for the two are equivalent.
\end{abstract}

\section{INTRODUCTION}

Recent work by Geng et al. in [1] identifies a broad regime where treating interference as noise (TIN) is optimal in the generalized degrees of freedom (GDoF) sense, and within a constant gap to exact capacity. Remarkably, the approximate optimality of TIN established in [1] is not only for sum-capacity but for the entire capacity region, and it fully incorporates power control. In fact power control is a crucial aspect of the TIN scheme, since it is generally not optimal for all nodes to transmit at full power if interference is treated as noise. Specifically, it is shown in [1] that in a $K$-user Gaussian interference channel, if for each user the desired signal strength is no less than the sum of the strengths of the strongest interference from this user and the strongest interference to this user (all values in $\mathrm{dB}$ scale), then TIN (with power control) is optimal from the perspective of GDoF and achieves the entire capacity region to within a constant gap.

In this work, we generalize the question of optimality of TIN to compound networks [2]-[5], with focus on interference channels. In a compound network, each receiver is associated with a set of states (a receiver's state is identified by the channel realizations associated with that receiver). The sets of possible states for each receiver are globally known apriori, however the transmitters are unaware of the particular realization chosen from within these sets. Thus, a reliable coding scheme must guarantee vanishing probability of error for every possible realization of each receiver from its given set of possible states. Equivalently, the compound interference channel can be described as having potentially multiple receivers for each message. This is known as the multiple

This work is supported in part by funding from ONR and by NSF grants CCF-1317351 and CCF-1319104. multicast, or multiple groupcast setting [6] and is of interest in its own right. An example of a 3-user compound interference channel is illustrated in Fig. 1.

Of particular interest for this work are compound networks where each possible state of the network individually satisfies the TIN-optimality condition of Geng et al. [1]. Is TIN still optimal for such a compound interference network as a whole, when all possible states are simultaneously considered? More generally, the implications of the compound setting on the GDoF region achievable through TIN (even if the TINoptimality conditions are not satisfied) is also of interest and will be explored in this work.

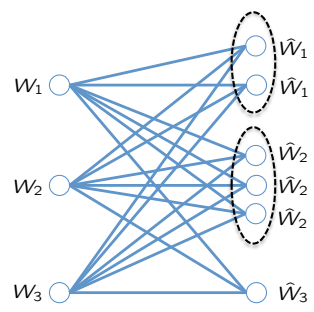

Fig. 1. A 3-user compound interference channel, where Receiver 1, 2, and 3 have 2, 3 and 1 possible states, respectively. For each receiver, the multiple potential states may also be interpreted as different users which intend to decode the common message from the corresponding transmitter. For this compound interference channel, there are totally $2 \times 3 \times 1=6$ possible network realizations (or states), each of which corresponds to a 3 -user regular interference channel.

\section{A. Challenges Posed by the Compound Setting}

Consider a $K$-user compound interference channel which satisfies the TIN-optimality condition of [1] in each possible state, individually. Recall that with the simple TIN scheme, each transmitter uses a point-to-point Gaussian codebook with an appropriate power level and each receiver only decodes the signal from its corresponding transmitter and treats the incoming interference as Gaussian noise. Denote by $\mathcal{P}$ the set of all the valid power allocations, $\mathcal{H}$ the set of all the possible network realizations, and $\mathcal{D}(\mathbf{P}, \mathbf{H})$ the achievable GDoF region through the TIN scheme for the network realization $\mathbf{H} \in \mathcal{H}$ with power allocation $\mathbf{P} \in \mathcal{P}$.

First, consider the achievability argument. Since the rate for each message in the TIN scheme is only limited by the minimum SINR (signal to interference and noise power ratio) 
across all states of the intended receiver, it is evident that for any valid power allocation $\mathbf{P} \in \mathcal{P}, \cap_{\mathbf{H} \in \mathcal{H}} \mathcal{D}(\mathbf{P}, \mathbf{H})$ is achievable. Taking the union of achievable rates over all the valid power allocations, we obtain the following inner bound on the GDoF region.

$$
\text { Inner Bound }=\cup_{\mathbf{P} \in \mathcal{P}} \cap_{\mathbf{H} \in \mathcal{H}} \mathcal{D}(\mathbf{P}, \mathbf{H})
$$

Next, consider the converse argument. Recall the assumption that the compound network satisfies the TIN-optimality condition of [1] in each possible state. In other words, each possible network state, by itself is TIN-optimal. For a given network state $\mathbf{H} \in \mathcal{H}$, since TIN is optimal, the entire GDoF region is achievable through power control and TIN, and it can be expressed as $\cup_{\mathbf{P} \in \mathcal{P}} \mathcal{D}(\mathbf{P}, \mathbf{H})$. Furthermore, since the GDoF region for each state is an outer bound for the GDoF region of the compound setting, by taking the intersection of the GDoF regions of all network states, we get the following outer bound on the GDoF region.

$$
\text { Outer Bound }=\cap_{\mathbf{H} \in \mathcal{H}} \cup_{\mathbf{P} \in \mathcal{P}} \mathcal{D}(\mathbf{P}, \mathbf{H})
$$

Note that while both the inner bound and the outer bound for the GDoF region involve union over power allocations and intersection over network states, the inner bound is the union of intersections whereas the outer bound is the intersection of unions. In general a union of intersections is (possibly strictly) smaller than or equal to an intersection of unions, consistent with their roles as inner and outer bounds, respectively. ${ }^{1}$ So the main challenge in settling the optimality of TIN for compound interference networks is to prove that, in our context, the two are indeed, identical.

\section{B. Main Contributions}

1) The optimality of TIN for compound interference channels: We show that for $K$-user compound Gaussian interference channels, if each possible network realization satisfies the TIN-optimality condition of [1], then power control and TIN achieves the entire GDoF region of the compound setting, which is the intersection of the GDoF regions of all possible network realizations. The result is derived from a non-trivial argument based on the potential theorem in [7], which builds upon the application of the potential theorem in [1]. For a general compound interference channel which may be not TIN-optimal, the entire TIN region (i.e., the achievable GDoF region through the TIN scheme) is also fully characterized.

2) Simplification of the compound interference channel into a regular counterpart: We show that for any $K$-user compound interference channel, regardless of the number of states for each receiver, we can always construct a counterpart $K$-user regular (where the network has only one state) interference channel, which has the same TIN region as that compound channel. Remarkably, the counterpart regular interference channel is in general none of the possible network

\footnotetext{
${ }^{1}$ Consider a simple example where $\mathcal{P}=\left\{\mathbf{P}_{\mathbf{1}}, \mathbf{P}_{\mathbf{2}}\right\}, \mathcal{H}=\left\{\mathbf{H}_{\mathbf{1}}, \mathbf{H}_{\mathbf{2}}\right\}$, $\mathcal{D}\left(\mathbf{P}_{\mathbf{1}}, \mathbf{H}_{\mathbf{1}}\right)=\{1\}, \mathcal{D}\left(\mathbf{P}_{\mathbf{1}}, \mathbf{H}_{\mathbf{2}}\right)=\{2\}, \mathcal{D}\left(\mathbf{P}_{\mathbf{2}}, \mathbf{H}_{\mathbf{1}}\right)=\{2\}$, and $\mathcal{D}\left(\mathbf{P}_{\mathbf{2}}, \mathbf{H}_{\mathbf{2}}\right)=\{1\}$. It is easy to check that the right hand sides of (1) and (2) are $\phi$ and $\{1,2\}$, respectively, which are not equal to each other.
}

realizations of the compound channel. In the full paper [8], we also show that to solve the GDoF-optimal power control problem for a $K$-user compound interference channel, we only need to solve the problem in its regular counterpart. In other words, from the GDoF perspective, the power control and TIN problems for compound and regular interference channels are equivalent, which significantly reduces the computational complexity of the GDoF-based power control in the compound setting. For instance, for the 3-user compound interference channel in Fig. 2(a), we can construct its counterpart 3-user regular interference channel, which is given in Fig. 2(b). Not only the two channels in Fig. 2(a) and 2(b) have the same TIN region, but also solving the GDoF-based power control problem for one is equivalent to solving the problem for the other. However, it should be noted that the compound channel and its regular counterpart are only equivalent in terms of the achievability of the TIN scheme. In [8], we give an example in which the compound interference channel is not TIN-optimal (i.e., there exist other achievable schemes outperforming the TIN scheme), while its regular counterpart satisfies the TINoptimality condition of [1].

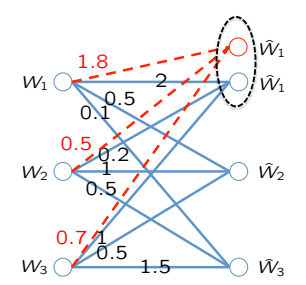

(a)

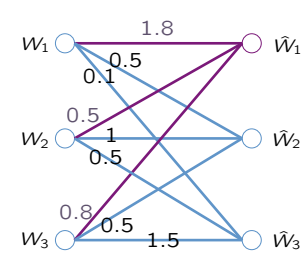

(b)
Fig. 2. (a) A 3-user compound interference channel, where Receiver 1 has two possible states. The value on each link denotes its channel strength level, which is defined in Section II formally; (b) The counterpart 3-user regular interference channel for the compound channel in Fig. 2(a).

Notations: Throughout this paper, for any positive integer $Z,\langle Z\rangle$ denotes the set $\{1,2, \ldots, Z\}$, and for any real number $a,(a)^{+}$and $\max \{0, a\}$ are used interchangeably. In addition, all logarithms are to the base 2 .

\section{Channel Model And Preliminaries}

Consider a $K$-user Gaussian interference channel consisting of $K$ transmitter-receiver pairs. The channel coefficients associated with Receiver $k, \forall k \in\langle K\rangle$, are denoted as a vector $\left(\tilde{h}_{k 1}, \tilde{h}_{k 2}, \ldots, \tilde{h}_{k K}\right)$, which is drawn from a finite set $\mathcal{L}_{k}$ with cardinality $L_{k}$. We assume that the channel coefficients remain fixed during the transmission. In addition, while the transmitters are unaware of the specific channel realizations, knowledge of $\mathcal{L}_{k}$ is assumed to be globally available. The receivers are assumed to have perfect channel state information. In this compound setting, reliable communication needs to be guaranteed simultaneously for all possible channel realizations. For Receiver $k$, the received signal in state $l_{k}$ is 
given by

$$
Y_{k}^{\left[l_{k}\right]}(t)=\sum_{i=1}^{K} \tilde{h}_{k i}^{\left[l_{k}\right]} \tilde{X}_{i}(t)+Z_{k}^{\left[l_{k}\right]}(t), \forall k \in\langle K\rangle, \forall l_{k} \in\left\langle L_{k}\right\rangle
$$

where $\tilde{h}_{k i}^{\left[l_{k}\right]}$ is the channel gain value from Transmitter $i$ to Receiver $k, \tilde{X}_{i}(t)$ and $Z_{k}^{\left[l_{k}\right]}(t)$ are the transmitted symbol of Transmitter $i$ and the additive white circularly symmetric Gaussian noise with zero mean and unit variance at Receiver $k$, respectively, in the $t$-th channel use. All symbols are complex. Each Transmitter $i$ is subject to the average power constraint $\mathbb{E}\left[\left|\tilde{X}_{i}(t)\right|^{2}\right] \leq P_{i}$.

To facilitate the GDoF studies, the standard channel model (3) is translated into an equivalent normalized form following similar approaches in [1], [9]. Define ${ }^{2}$

$$
\alpha_{k i}^{\left[l_{k}\right]} \triangleq \frac{\log \left(\max \left\{1,\left|\tilde{h}_{k i}^{\left[l_{k}\right]}\right|^{2} P_{i}\right\}\right)}{\log P}, \quad \forall i, k \in\langle K\rangle, \forall l_{k} \in\left\langle L_{k}\right\rangle
$$

where $P>1$ is a nominal power value. Then the original channel model (3) can be presented in the following form,

$$
\begin{aligned}
Y_{k}^{\left[l_{k}\right]}(t)= & \sum_{i=1}^{K} \sqrt{P^{\alpha_{k i}^{\left[l_{k}\right]}}} e^{j \theta_{k i}^{\left[l_{k}\right]}} X_{i}(t)+Z_{k}^{\left[l_{k}\right]}(t), \\
& \forall k \in\langle K\rangle, \forall l_{k} \in\left\langle L_{k}\right\rangle
\end{aligned}
$$

where $X_{i}(t)$ is the normalized transmit symbol of Transmitter $i$, subject to the unit power constraint, i.e., $\mathbb{E}\left[\left|X_{i}(t)\right|^{2}\right] \leq 1$. $\sqrt{P^{\alpha_{k i}^{\left[l_{k}\right]}}}$ and $\theta_{k i}^{\left[l_{k}\right]}$ are the channel magnitude and phase between Transmitter $i$ and Receiver $k$ under state $l_{k}$, respectively. As in [1], $\alpha_{k i}^{\left[l_{k}\right]}$ is called the channel strength level. In the rest of this paper we will consider the equivalent channel model in (4). The definitions of messages, achievable rates, capacity region, generalized degrees of freedom $(\mathrm{GDoF})$ are all standard (see, e.g., [1], [8]) and thus omitted here.

\section{A. The Achievable GDoF Region through TIN}

In the $K$-user compound interference channel, we assume the power allocated to Transmitter $k$ is $P^{r_{k}}$. Due to the unit power constraint, $r_{k} \leq 0, \forall k \in\langle K\rangle$. The rate achievable by User $k$ through TIN is limited by the smallest SINR across all states possible for this user. So User $k \in\langle K\rangle$ can achieve any rate $R_{k}$ such that

$$
R_{k} \leq \min _{l_{k}}\left\{\log \left(1+\frac{P^{r_{k}} \times P^{\alpha_{k k}^{\left[l_{k}\right]}}}{1+\sum_{j=1, j \neq k}^{K} P^{r_{j}} \times P^{\alpha_{k j}^{\left[l_{k}\right]}}}\right)\right\}
$$

In the GDoF sense, we have

$$
0 \leq d_{k} \leq \min _{l_{k}}\left\{\max \left\{0, \alpha_{k k}^{\left[l_{k}\right]}+r_{k}-\left(\max _{j: j \neq k}\left\{\alpha_{k j}^{\left[l_{k}\right]}+r_{j}\right\}\right)^{+}\right\}\right\}
$$

The TIN region, which is denoted by $\mathcal{P}^{*}$, is the set of all GDoF tuples $\left(d_{1}, d_{2}, \ldots, d_{K}\right)$ for which there exist $r_{k}$ 's, such

\footnotetext{
${ }^{2}$ Similar to [1], it is not hard to verify that avoiding negative $\alpha$ 's has no impact on the GDoF results.
}

that $\forall k \in\langle K\rangle$

$$
\begin{aligned}
& r_{k} \leq 0, \\
& d_{k} \geq 0, \\
& d_{k} \leq \min _{l_{k}}\left\{\max \left\{0, \alpha_{k k}^{\left[l_{k}\right]}+r_{k}-\left(\max _{j: j \neq k}\left\{\alpha_{k j}^{\left[l_{k}\right]}+r_{j}\right\}\right)^{+}\right\}\right\}
\end{aligned}
$$

Similar to [1], we also introduce a polyhedral version of the TIN scheme, which is called the polyhedral TIN scheme. By requiring $\min _{l_{k}}\left\{\alpha_{k k}^{\left[l_{k}\right]}+r_{k}-\left(\max _{j: j \neq k}\left\{\alpha_{k j}^{\left[l_{k}\right]}+r_{j}\right\}\right)^{+}\right\}$to be no less than 0 for all users, we can ignore the first $\max \{0,$. term in (6) and obtain

$$
0 \leq d_{k} \leq \min _{l_{k}}\left\{\alpha_{k k}^{\left[l_{k}\right]}+r_{k}-\left(\max _{j: j \neq k}\left\{\alpha_{k j}^{\left[l_{k}\right]}+r_{j}\right\}\right)^{+}\right\}
$$

We call the new achievable GDoF region after the above modification the polyhedral TIN region $\mathcal{P}$. So $\mathcal{P}$ is the set of all GDoF tuples $\left(d_{1}, d_{2}, \ldots, d_{K}\right)$ for which there exist $r_{k}$ 's, $k \in\langle K\rangle$, such that the equations (8)-(11) at the top of next page are satisfied.

In general, with this modification we require that the right hand side of (7) is non-negative for all users $k \in\langle K\rangle$, hence we put more constraints on the power exponents $r_{i}$ 's besides the constraints of $r_{i} \leq 0$, which can only shrink the achievable GDoF region via power control and TIN. In other words, $\mathcal{P} \subseteq$ $\mathcal{P}^{*}$. Later our results will show that when certain conditions hold, $\mathcal{P}=\mathcal{P}^{*}$, i.e., the above modification incurs no loss.

\section{RESULTS}

In this section, we present the results of this work. ${ }^{3}$

The following theorem provides a broadly applicable condition under which power control and TIN achieves the entire GDoF region of a $K$-user compound interference channel.

Theorem 1: In a $K$-user compound interference channel, if the following condition is satisfied,

$$
\begin{gathered}
\alpha_{i i}^{\left[l_{i}\right]} \geq \max _{j: j \neq i}\left\{\alpha_{j i}^{\left[l_{j}\right]}\right\}+\max _{k: k \neq i}\left\{\alpha_{i k}^{\left[l_{i}\right]}\right\}, \\
\forall i, j, k \in\langle K\rangle, \forall l_{i} \in\left\langle L_{i}\right\rangle, \forall l_{j} \in\left\langle L_{j}\right\rangle
\end{gathered}
$$

then power control and TIN achieves the entire GDoF region, which is the intersection of the GDoF regions of all the possible network realizations and includes all the GDoF tuples $\left(d_{1}, d_{2}, \ldots, d_{K}\right)$ satisfying

$$
\begin{gathered}
0 \leq d_{i} \leq \alpha_{i i}^{\left[l_{i}\right]}, \quad \forall i \in\langle K\rangle, \forall l_{i} \in\left\langle L_{i}\right\rangle \\
\sum_{j=1}^{m} d_{i_{j}} \leq \sum_{j=1}^{m}\left(\alpha_{i_{j} i_{j}}^{\left[l_{i_{j}}\right]}-\alpha_{i_{j} i_{j+1}}^{\left[l_{i_{j}}\right]}\right) \\
\forall\left(i_{1}, \ldots, i_{m}\right) \in \Pi_{K}, \forall m \in\{2,3, \ldots, K\}, \forall l_{i_{j}} \in\left\langle L_{i_{j}}\right\rangle
\end{gathered}
$$

where $\Pi_{K}$ is the set of all possible cyclic sequences ${ }^{4}$ of all subsets of $\langle K\rangle$ with cardinality no less than 2 , and the modulo$m$ arithmetic is implicitly used on user indices, e.g., $i_{m}=i_{0}$.

\footnotetext{
${ }^{3}$ Readers can refer to illustrative examples and remarks in the full paper [8], which help understand the results of this work.

${ }^{4}$ Each cyclic sequence in $\Pi_{K}$ is a cyclically ordered subset of user indices, without repetitions. For instance, $\Pi_{3}=$ $\{(1,2),(1,3),(2,3),(1,2,3),(1,3,2)\}$.
} 


$$
\begin{aligned}
r_{k} & \leq 0, & & \forall k \in\langle K\rangle \\
d_{k} & \geq 0, & & \forall k \in\langle K\rangle \\
d_{k} \leq \alpha_{k k}^{\left[l_{k}\right]}+r_{k} \Leftrightarrow r_{k} & \geq d_{k}-\alpha_{k k}^{\left[l_{k}\right],} & & \forall k \in\langle K\rangle, \forall l_{k} \in\left\langle L_{k}\right\rangle \\
d_{k} \leq \alpha_{k k}^{\left[l_{k}\right]}+r_{k}-\left(\alpha_{k j}^{\left[l_{k}\right]}+r_{j}\right) \Leftrightarrow r_{k}-r_{j} & \geq\left(\alpha_{k j}^{\left[l_{k}\right]}-\alpha_{k k}^{\left[l_{k}\right]}\right)+d_{k}, & & \forall k, j \in\langle K\rangle, k \neq j, \forall l_{k} \in\left\langle L_{k}\right\rangle
\end{aligned}
$$

Proof: For the converse, we first consider each possible network state individually. Since under the condition (12), in each network state, the channel satisfies the TIN-optimality condition identified in [1], it is not hard to characterize its GDoF region. Taking the intersection of the GDoF regions of all the possible network states, we have the desired outer bounds.

Next, consider the achievability. Recall that for the $K$-user compound interference channel, the polyhedral TIN region $\mathcal{P}$ defined in Section II-A is characterized by (8)-(11). Setting

$$
r_{k}^{\left[l_{k}\right]}=r_{k}, \quad \forall k \in\langle K\rangle, \forall l_{k} \in\left\langle L_{k}\right\rangle,
$$

it is easy to check that the set of the inequalities (8)-(11) is equivalent to (16)-(20) given at the top of next page. In other words, for a GDoF tuple $\left(d_{1}, d_{2}, \ldots, d_{K}\right) \in \mathbb{R}_{+}^{K}$ (i.e., the nonnegative orthant of the $K$-dimensional Euclidean space), it is in the polyhedral TIN region $\mathcal{P}$ if and only if there exists $r_{k}^{\left[l_{k}\right]}$, $k \in\langle K\rangle, l_{k} \in\left\langle L_{k}\right\rangle$, such that (17)-(20) hold.

Then for any $K$-user compound interference channel, we construct a complete digraph $D_{p}=(V, E)$ with $\sum_{k} L_{k}+1$ vertices, where $V$ and $E$ are the sets of vertices and edges, respectively, and

$$
V=\left\{v_{1}^{[1]}, \ldots, v_{k}^{\left[l_{k}\right]}, \ldots, v_{K}^{\left[L_{K}\right]}, u\right\}, \forall k \in\langle K\rangle, \forall l_{k} \in\left\langle L_{k}\right\rangle
$$

We assign a length $l(e)$ to each edge $e \in E$ following (21)(24) in next page, where $(a, b)$ denotes the edge from vertex $a$ to vertex $b$. We call such a digraph $D_{p}$ the potential graph for the $K$-user compound interference channel. As an example, the potential graph $D_{p}$ for a 2-user compound interference channel with $L_{1}=2$ and $L_{2}=1$ is given in Fig. 3 .

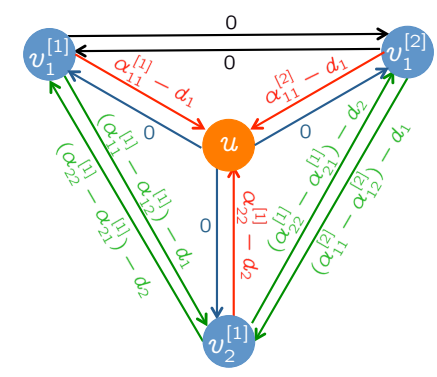

Fig. 3. The potential graph $D_{p}$ for a 2-user compound interference channel with $L_{1}=2$ and $L_{2}=1$, where the value on each edge denotes the edge length.

By definition [7], for a graph a function $p: V \rightarrow \mathbb{R}$ is called a potential if for every two vertices $a, b \in V$ such that $(a, b) \in E, l(a, b) \geq p(b)-p(a)$. Note these inequalities depend upon the difference between potential function values only. Therefore, without loss of generality, if there exists a valid potential function for the potential graph $D_{p}$, we can make the vertex $u$ ground, i.e., $p(u)=0$. Then let $r_{k}^{\left[l_{k}\right]}:=p\left(v_{k}^{\left[l_{k}\right]}\right), \forall k \in\langle K\rangle, \forall l_{k} \in\left\langle L_{k}\right\rangle$. It is not hard to check that the potential function values should satisfy the inequalities (17)-(20). In other words, in a $K$-user compound interference channel, for a GDoF tuple $\left(d_{1}, d_{2}, \ldots, d_{K}\right) \in \mathbb{R}_{+}^{K}$, it is in the region $\mathcal{P}$ if and only if there exists a valid potential function for its potential graph $D_{p}$.

Finally, based on the potential theorem in [7], which says that there exists a potential function for a digraph $D$ if and only if each directed circuit in $D$ has a non-negative length, we conclude that for a GDoF tuple $\left(d_{1}, d_{2}, \ldots, d_{K}\right) \in \mathbb{R}_{+}^{K}$, it is in the region $\mathcal{P}$ if and only if the length of each directed circuit in $D_{p}$ is non-negative. Therefore, to characterize the polyhedral TIN region $\mathcal{P}$, the only job left is to make sure the lengths of all the directed circuits in $D_{p}$ are no less than 0 . We categorize all the directed circuits of $D_{p}$ into the following three classes:

- Circuits in the form of $\left(u \rightarrow v_{k}^{\left[l_{k}\right]} \rightarrow u\right)$. For these circuits, the non-negative length condition gives us

$$
\alpha_{k k}^{\left[l_{k}\right]}-d_{k} \geq 0 \Leftrightarrow d_{k} \leq \alpha_{k k}^{\left[l_{k}\right]}
$$

- Circuits in the form $\left(v_{i_{0}}^{\left[l_{i_{0}}\right]} \rightarrow v_{i_{1}}^{\left[l_{i_{1}}\right]} \rightarrow \ldots \rightarrow v_{i_{m}}^{\left[l_{i_{m}}\right]}\right)$, where $i_{0}=i_{m}$ and $\left(i_{1}, i_{2}, \ldots, i_{m}\right) \in \Pi_{K}$. For these circuits, the non-negative length condition becomes

$$
\begin{array}{r}
\sum_{j=1}^{m}\left(\alpha_{i_{j} i_{j}}^{\left[l_{i_{j}}\right]}-\alpha_{i_{j} i_{j+1}}^{\left[l_{i_{j}}\right]}-d_{i_{j}}\right) \geq 0 \\
\Leftrightarrow \sum_{j=1}^{m} d_{i_{j}} \leq \sum_{j=1}^{m}\left(\alpha_{i_{j} i_{j}}^{\left[l_{i_{j}}\right]}-\alpha_{i_{j} i_{j+1}}^{\left[l_{i_{j}}\right]}\right)
\end{array}
$$

- All the other circuits. For the remaining circuits, it is easy to check that given (25) and (26), the inequalities derived from the non-negative length condition are all redundant.

Consequently, we end up with the conditions (25)-(26). Explicitly adding the non-negative constraint on $d_{i}$ 's in (25)(26), we obtain the polyhedral TIN region $\mathcal{P}$, which turns out to be the intersection of the polyhedral TIN regions for all the possible network realizations and fully characterized by (13)(14). Clearly, under condition (12), the polyhedral TIN region $\mathcal{P}$ coincides with the derived outer bounds. Thus we complete the proof.

Based on the result of Theorem 1, we further obtain the TIN region $\mathcal{P}^{*}$ for general $K$-user compound interference 


$$
\begin{aligned}
d_{k} & \geq 0, & & \forall k \in\langle K\rangle \\
r_{k}^{\left[l_{k}\right]} & \leq 0, & & \forall k \in\langle K\rangle, \forall l_{k} \in\left\langle L_{k}\right\rangle \\
r_{k}^{\left[l_{k}\right]}-r_{k}^{\left[l_{k}^{\prime}\right]} & \leq 0, & & \forall k \in\langle K\rangle, \forall l_{k}, l_{k}^{\prime} \in\left\langle L_{k}\right\rangle, l_{k} \neq l_{k}^{\prime} \\
r_{k}^{\left[l_{k}\right]} & \geq d_{k}-\alpha_{k k}^{\left[l_{k}\right]}, & & \forall k \in\langle K\rangle, \forall l_{k} \in\left\langle L_{k}\right\rangle \\
r_{k}^{\left[l_{k}\right]}-r_{j}^{\left[l_{j}\right]} & \geq\left(\alpha_{k j}^{\left[l_{k}\right]}-\alpha_{k k}^{\left[l_{k}\right]}\right)+d_{k}, & & \forall k, j \in\langle K\rangle, k \neq j, \forall l_{k} \in\left\langle L_{k}\right\rangle, \forall l_{j} \in\left\langle L_{j}\right\rangle
\end{aligned}
$$

$$
\begin{aligned}
l\left(v_{k}^{\left[l_{k}\right]}, v_{k}^{\left[l_{k}^{\prime}\right]}\right) & =0, & & \forall k \in\langle K\rangle, \forall l_{k}, l_{k}^{\prime} \in\left\langle L_{k}\right\rangle, l_{k} \neq l_{k}^{\prime} \\
l\left(v_{k}^{\left[l_{k}\right]}, v_{j}^{\left[l_{j}\right]}\right) & =\left(\alpha_{k k}^{\left[l_{k}\right]}-\alpha_{k j}^{\left[l_{k}\right]}\right)-d_{k}, & & \forall k, j \in\langle K\rangle, k \neq j, \forall l_{k} \in\left\langle L_{k}\right\rangle, \forall l_{j} \in\left\langle L_{j}\right\rangle \\
l\left(v_{k}^{\left[l_{k}\right]}, u\right) & =\alpha_{k k}^{\left[l_{k}\right]}-d_{k}, & & \forall k \in\langle K\rangle, \forall l_{k} \in\left\langle L_{k}\right\rangle \\
l\left(u, v_{k}^{\left[l_{k}\right]}\right) & =0, & & \forall k \in\langle K\rangle, \forall l_{k} \in\left\langle L_{k}\right\rangle
\end{aligned}
$$

channels. Following along the lines of Theorem 5 in [1], we get that in general $\mathcal{P}^{*}$ is a union of $2^{K}$ polyhedral TIN regions $\mathcal{P}_{\mathcal{S}}$, each of which corresponds to the case where the users in $\mathcal{S}$ (any subset of $\langle K\rangle$ ) are deactivated and thus removed from the network. Note that for general compound interference channels, $\mathcal{P}_{\phi}$ (i.e., the polyhedral TIN region for the case in which all the users are active) is the same as the polyhedral TIN region $\mathcal{P}$ defined in Section II-A, i.e., $\mathcal{P}=\mathcal{P}_{\phi}$. When (12) is satisfied, the polyhedral TIN region $\mathcal{P}_{\phi}$ subsumes all the others and $\mathcal{P}^{*}=\mathcal{P}$.

In the sequel, we show how to construct a regular (i.e., not compound) interference channel that has the same TIN region as a given compound interference channel where TIN may or may not be optimal. Denote the $K$-user compound interference channel defined in (4) as $\mathcal{I C}_{C}$. Based on $\mathcal{I C}_{C}$, we construct a counterpart $K$-user regular interference channel $\mathcal{I C}_{R}$ with the input-output relationship

$$
Y_{k}(t)=\sum_{i=1}^{K} \sqrt{P^{\bar{\alpha}_{k i}}} e^{j \theta_{k i}} X_{i}(t)+\bar{Z}_{k}(t), \quad \forall k \in\langle K\rangle,
$$

where

$$
\begin{array}{ll}
\bar{\alpha}_{k k}=\min _{l_{k}}\left\{\alpha_{k k}^{\left[l_{k}\right]}\right\}, & \forall k \in\langle K\rangle \\
\bar{\alpha}_{k j}=\min _{l_{k}}\left\{\alpha_{k k}^{\left[l_{k}\right]}\right\}-\min _{l_{k}}\left\{\alpha_{k k}^{\left[l_{k}\right]}-\alpha_{k j}^{\left[l_{k}\right]}\right\}, & \forall k, j \in\langle K\rangle, j \neq k,
\end{array}
$$

and $\bar{Z}_{k}(t) \sim \mathcal{C N}(0,1)$. It is noteworthy that the regular interference channel $\mathcal{I C}_{R}$ is a non-trivial mixture of states of the compound channel $\mathcal{I C}_{C}$.

The following theorem states the equivalence result.

Theorem 2: The $K$-user compound interference channel $\mathcal{I C}_{C}$ and its counterpart $K$-user regular interference channel $\mathcal{I C}_{R}$ have the same TIN region $\mathcal{P}^{*}$ and the same polyhedral TIN region $\mathcal{P}$.

The proof details of Theorem 2 are deferred to [8]. The equivalence is particularly useful for GDoF-optimal power control algorithms for compound channels. In [8], taking advantage of the simplification of the compound setting to the regular case and using additional insights from the potential theorem, we devise a centralized iterative power control algorithm, which requires at most $K$ updates, to obtain the optimal power allocation for any feasible GDoF tuple of general $K$ user compound interference channels.

\section{CONCLUSION}

We show that for a $K$-user compound Gaussian interference channel, if in each possible network state, the channel satisfies the TIN-optimality condition identified in [1], then its GDoF region is the intersection of the GDoF regions of all the network states, which is achievable via power control and TIN. We also demonstrate how to construct, for a general compound interference channel, a counterpart regular interference channel that has the same TIN region, which leads to a simple solution of the GDoF-optimal power control problem for compound settings.

\section{REFERENCES}

[1] C. Geng, N. Naderializadeh, A. S. Avestimehr, and S. A. Jafar, "On the optimality of treating interference as noise," IEEE Transactions on Information Theory, vol. 61, no. 4, pp. 1753-1767, Apr. 2015.

[2] H. Weingarten, S. Shamai, and G. Kramer, "On the compound MIMO broadcast channel," presented at the Information Theory and Application Workshop, Jan. 2007.

[3] T. Gou, S. A. Jafar, and C. Wang, "On the degrees of freedom of finite state compound wireless networks," IEEE Transactions on Information Theory, vol. 57, no. 6, pp. 3286-3308, June 2011.

[4] M. A. Maddah-Ali, "On the degrees of freedom of the compound MIMO broadcast channels with finite states," e-print arXiv: 0909.5006v3, Oct. 2009. Avaliable at http://arxiv.org/abs/0909.5006.

[5] A. Raja, V. M. Prabhakaran, and P. Viswanath, "The two-user compound interference channel," IEEE Transactions on Information Theory, vol. 55, no. 11, pp. 5100-5120, Nov. 2009.

[6] H. Maleki, V. R. Cadambe, and S. A. Jafar, "Index coding - an interference alignment perspective," IEEE Transactions on Information Theory, vol. 60, no. 9, pp. 5402-5432, Sep. 2014.

[7] A. Schrijver, Combinatorial Optimization, Springer, 2003.

[8] C. Geng, and S. A. Jafar, "On the optimality of treating interference as noise: compound interference networks," e-print Arxiv:1412.2711, Dec. 2014. Avaliable at http://arxiv.org/abs/1412.2711.

[9] R. Etkin, D. Tse, and H. Wang, "Gaussian interference channel capacity to within one bit," IEEE Transactions on Inforamtion Thoery, vol. 54, no. 12, pp. 5534-5562, Dec. 2008. 